INPLASY

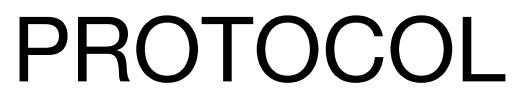

To cite: Wang et al. The efficacy of median nerve electrical stimulation on recovery of patients with consciousness disorders - a meta-analysis. Inplasy protocol 202170037. doi:

10.37766/inplasy2021.7.0037

Received: 12 July 2021

Published: 12 July 2021

Corresponding author:

Peng Wang

wpsdslyy@hotmail.com

Author Affiliation:

Shandong Provincial Hospital Affiliated to Shandong First Medical University.

Support: 2020M143.

Review Stage at time of this submission: The review has not yet started.

Conflicts of interest:

None declared.

\section{The efficacy of median nerve electrical stimulation on recovery of patients with consciousness disorders - a meta-analysis}

Wang, P1; Zhou, H²; Zhang, H³; Zhang, L4; Sui, Y5; Zhang, Z6.

Review question / Objective: Whether median nerve electrical stimulation can promote the recovery of patients with consciousness disorders.

Condition being studied: Consciousness disorder.

Information sources: At minimum, we will search the following databases: PubMed, Cochrane Central Register of Controlled trials, the China National Knowledge Infrastructure(CNKI), Chinese VIP Information(VIP), Wanfang. We will hand search bibliographies from systematic reviews and meta-analyses on the topic.

INPLASY registration number: This protocol was registered with the International Platform of Registered Systematic Review and Meta-Analysis Protocols (INPLASY) on 12 July 2021 and was last updated on 12 July 2021 (registration number INPLASY202170037).

\section{INTRODUCTION}

Review question / Objective: Whether median nerve electrical stimulation can promote the recovery of patients with consciousness disorders.

Condition being studied: Consciousness disorder.

\section{METHODS}

Participant or population: Patients with consciousness disorders caused by any reasons.

Intervention: Median nerve stimulation.

Comparator: Any conventional methods. 
Study designs to be included: Random control clinical trials.

Eligibility criteria: Any study detailing median nerve stimulation and its use for patients with consciousness disorders.

Information sources: At minimum, we will search the following databases: PubMed, Cochrane Central Register of Controlled trials, the China National Knowledge Infrastructure(CNKI), Chinese VIP Information (VIP), Wanfang. We will hand search bibliographies from systematic reviews and meta-analyses on the topic.

Main outcome(s): 1.Glasgow Coma Scale.

Additional outcome (s): 1 . Electroencephalogram 2. Brainstem evoked potential 3. Improvement of movement or sensory funcyion.

Quality assessment / Risk of bias analysis: Selected studies will be critically appraised by two independent reviewers at the study level for methodological quality in the review using the standardized critical appraisal instruments, The Cochrane Collaboration's tool for assessing risk of bias. Any disagreements that arise will be resolved through discussion, or with a third individual.

Strategy of data synthesis: Papers will be pooled in statistical meta-analysis using Revman 5. Effect sizes will be expressed as either odds ratios (for dichotomous data and weighted (or standardized) mean differences (for continuous data) and their 95\% confidence intervals will be calculated for analysis Modify as appropriate. Heterogeneity will be assessed statistically using the Cochran $Q$ test and I squared tests. A funnel plot will be generated to assess publication bias if there are 10 or more studies included in a meta-analysis. Statistical tests for funnel plot asymmetry (Egger test, Begg test, Harbord test) will be performed where appropriate.

Subgroup analysis: If there is significant heterogeneity in the results, we will conduct subgroup analysis (such as characteristics of study participants, treatment duration and different types of treatment), subgroup analysis would be applied to identify the source of heterogeneity.

Sensitivity analysis: The sensitivity analysis will be performed to test the stability of the results of meta-analysis by removing the low quality studies.

Country(ies) involved: China.

Keywords: Consciousness disorder; Median nerve electrical stimulation; Metaanalysis.

Contributions of each author:

Author 1 - Peng Wang.

Author 2 - Hong Zhou.

Author 3 - Huanxin Zhang.

Author 4 - Lunzhong Zhang.

Author 5 - Yunlong Sui.

Author 6 - Zhen Zhang. 